\title{
Synthesis of $\mathrm{MoO}_{3}$ and its polyvinyl alcohol nanostructured film
}

\author{
ARUNKUMAR LAGASHETTY ${ }^{\dagger}$, VIJAYANAND HAVANOOR, S BASAVARAJA and \\ A VENKATARAMAN* \\ Department of Materials Science and Chemistry, Gulbarga University, Gulbarga 585 106, India \\ ${ }^{\dagger}$ Appa Institute of Engineering and Technology, Gulbarga 585 101, India
}

MS received 9 December 2004

\begin{abstract}
The synthesis of ultrafine $\mathrm{MoO}_{3}$ through a self-propagating combustion route employing polyethylene glycol as fuel is reported. The precursor molybdenum oxalate is employed in this study for the conversion of the precursor to ultrafine $\mathrm{MoO}_{3}$ particles. The solvent casting method is adopted for the synthesis of $\mathrm{MoO}_{3}$ dispersed polyvinyl alcohol nanostructured film $\left(\mathrm{MoO}_{3}-\mathrm{PVA}\right)$. These synthesized $\mathrm{MoO}_{3}$ and their composite samples are characterized for their structure, morphology, bonding and thermal behaviour by XRD, SEM, IR and DSC techniques, respectively. The distribution of $\mathrm{MoO}_{3}$ in polyvinyl alcohol gives a crystalline polymer, a compact structure and an increase in glass transition temperature.
\end{abstract}

Keywords. Combustion method; $\mathrm{MoO}_{3}$; polyvinyl alcohol; nanocomposite.

\section{Introduction}

The research in new synthetic route for the nanoceramics is an integral aspect of material chemistry (Rao 1994). In recent times, development of different synthetic techniques such as soft chemical and sol-gel methods have led to the engineering materials (Gopalakrishnan 1995). Now a days microwave assisted synthetic route is becoming a very rapidly developed method (Rao et al 1999a,b; Harish Bhat et al 2000; ChungChen and Huang 2002; Lagashetty et al 2002). New Chimie-Douce routes for the synthesis of cobalt ferrite and other ceramics are being continuously investigated (Figlarz 1989; Lee et al 1998; Helen and Kamath 2000). In our earlier work, we have reported the combustion synthesis of nanoparticles using polymer as a fuel (Lagashetty et al 2003; Mallikarjuna et al 2003a,b). These combustion derived nanoparticles act as good adsorbent for heavier metal ions like lead ions (Mallikarjuna and Venkataraman 2003). The adsorption of lead ions on nanoparticles is increased by complexing with thiourea as a ligand (Lagashetty et al 2003; Mallikarjuna et al 2003a,b).

Polymer nanocomposites, also presently known as 'nanostructured materials', are materials in which nanoscopic inorganic particles, typically $10-100 \mathrm{~nm}$ in atleast one dimension, are dispersed in an organic polymer matrix in order to dramatically improve the performance properties of the polymer (Mzdujic et al 1998; Jungk and Feldmann 2000; Richard et al 2000). Nanostructured

*Author for correspondence (raman_chem@ $@$ rediffmail.com) materials based on nanosized ceramic have been of great interest to researchers due to their possible applications in refrigeration and high-density information storage (Shull and Bennett 1992; Xiao et al 1993; Sinha 2002). These composites are often prepared by dispersing ceramic materials in a polymer matrix (Castro et al 2000; Cheng et al 2000; Yurekli et al 2001).

In this paper we report the synthesis of an important layered ceramic material, $\mathrm{MoO}_{3}$, through a self-propagating combustion route. This synthetic route can be considered interesting for its simplicity, reproducibility and easy scale up. It is a low-energy reaction and can be carried out in a china dish in an open atmosphere. In this self-propagation combustion reaction, a suitable fuel was found to be poly (ethylene glycol). In our earlier report, we had employed reduction of ammonium molybdate (Mallikarjuna and Venkataraman 2001) and decomposition of molybdate gel (Lagashetty et al 2003; Mallikarjuna et al 2003a,b) to obtain ultrafine dimensions of $\mathrm{MoO}_{3}$. This work is an extension of our earlier work on the feasibility of employing new synthetic route to the layered materials. The formation of nanostructured materials with interesting electrical, magnetic and thermal properties are obtained when nanoscale metal oxides are incorporated into the polymer matrix (Mallikarjuna et al 2004; Govindraj et al 2004a,b). We also report the solvent casting method for the synthesis of $\mathrm{MoO}_{3}-\mathrm{PVA}$ composite film. The as prepared $\mathrm{MoO}_{3}$ nanoparticles and their nanostructured materials are characterized for their structure employing spectroscopic (infrared), X-ray diffraction (XRD), scanning electron micrograph (SEM) and thermal (DSC) techniques. 


\section{Experimental}

\subsection{Material and methods}

Ammonium molybdate and oxalic acid employed were of AR grades. Polyethylene glycol (PEG) having molecular weight 4000 and polyvinyl alcohol (PVA) were obtained commercially from M/s Himedia chemicals, Mumbai. Oxygen free double distilled water was used in the present work. The combustion method was adopted for the synthesis of $\mathrm{MoO}_{3}$ and solvent casting method was adopted for the synthesis of $\mathrm{MoO}_{3}$-PVA composite.

\subsection{Preparation of molybdenum oxalate}

A mixture of equimolar solutions of ammonium molybdate and oxalic acid was stirred well. The precipitate of molybdenum oxalate obtained was filtered through sintered glass crucible and washed with cold distilled water and finally washed with dry acetone and then dried under vacuum.

\subsection{Synthesis of nanosized $\mathrm{MoO}_{3}$}

The above prepared molybdenum oxalate was mixed with polyethylene glycol in the weight ratio $1: 5$ and ground well in a pestle and mortar (Venkataraman et al 2001). The resultant solid was placed in a crucible and heated in presence of atmospheric air. It was observed that initially polyethylene glycol melted, then frothed and finally ignited to form $\mathrm{MoO}_{3}$. On cooling to room temperature no trace of carbon impurities was observed in the $\mathrm{MoO}_{3}$ residue. $\mathrm{XRD}, \mathrm{SEM}$ and IR studies characterized the synthesized $\mathrm{MoO}_{3}$.

\subsection{Synthesis of $\mathrm{MoO}_{3}$ dispersed polyvinyl alcohol ( $\mathrm{MoO}_{3}-\mathrm{PVA}$ ) nanostructured film}

The method employed for the synthesis of $\mathrm{MoO}_{3}-\mathrm{PVA}$ composite was a solvent casting method. A known weight ( $4 \mathrm{~g}$ ) of polyvinyl alcohol was dissolved in double distilled water and required amount $(0 \cdot 1 \mathrm{~g})$ of $\mathrm{MoO}_{3}$ (10 wt $\%)$ was added and stirred well in a magnetic hot plate. The whole mass was transferred to a clean petridish and kept in a vacuum desiccator for complete evaporation of water. A well dispersed thin film of $\mathrm{MoO}_{3}$ inserted PVA was obtained. The prepared film was free from air bubbles and with uniformly dispersed $\mathrm{MoO}_{3}$ particles. This nanostructured material was then characterized by XRD, SEM, IR and thermal techniques.

\subsection{Characterization}

The powder X-ray diffraction pattern was obtained from GEOL JDX-8P X-ray diffractometer using $\operatorname{CoK} \alpha$ radia- tion. The morphology of the ceramics as well as polymer nanostructured film were obtained from Leica Cambridge-440 scanning electron microscope. The infrared spectra of nanoceramics were recorded on a Perkin-Elmer FTIR spectrometer [model 100] in the range 4000$300 \mathrm{~cm}^{-1}$. Thermal traces were obtained from Mettler Toledo Star instrument.

\section{Results and discussion}

\subsection{X-ray diffraction}

Figure 1 shows the XRD pattern of as synthesized $\mathrm{MoO}_{3}$. This sample produces a diffraction pattern with large number of peaks. The $d$-spacing values of the sample match well with the standard $\mathrm{MoO}_{3}$ file (JCPDS 5-508). Figure 2 shows the XRD pattern of pure polyvinyl alcohol. The pattern shows no intense peaks throughout the spectrum indicating amorphous nature of the sample.

Figure 3 shows the XRD pattern of as synthesized $\mathrm{MoO}_{3}$-PVA nanostructured film. Sharp and highly intense peaks are observed. Some additional sharp peaks are also observed when compared with pure polyvinyl alcohol (figure 2). We also observe some $\mathrm{MoO}_{3}$ peaks in the composite spectrum. On comparison with figures 1 and 2 , the formation of partially crystalline nanostructured film is understood.

\subsection{Scanning electron micrograph}

The surface morphology of as synthesized $\mathrm{MoO}_{3}$ and its nanostructured film is studied by scanning electron micrograph images. Figures 4a,b show the SEM images of as synthesized $\mathrm{MoO}_{3}$ under low and high resolution, respectively. From these images various shapes and sizes of the particles are observed. There is aggregation of particles as well as particle agglomerates. However, under high resolution formation of a compact structure is observed.

Figures 5a,b show the SEM images of as synthesized $\mathrm{MoO}_{3}$-PVA nanostructured film at low and high magni-

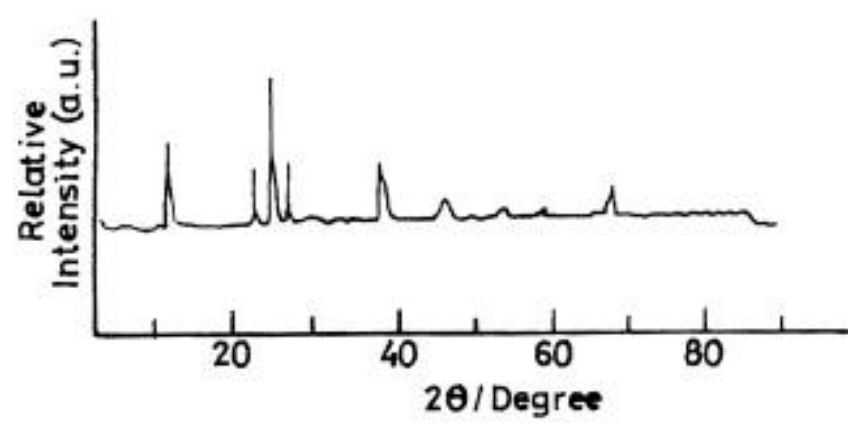

Figure 1. XRD pattern of as synthesized $\mathrm{MoO}_{3}$. 
fication, respectively. From this image it is observed that polyvinyl alcohol has irregular shaped $\mathrm{MoO}_{3}$ particles dispersed in its matrix. However, on high resolution clear fine dispersion of $\mathrm{MoO}_{3}$ particle in the polymer matrix is observed.

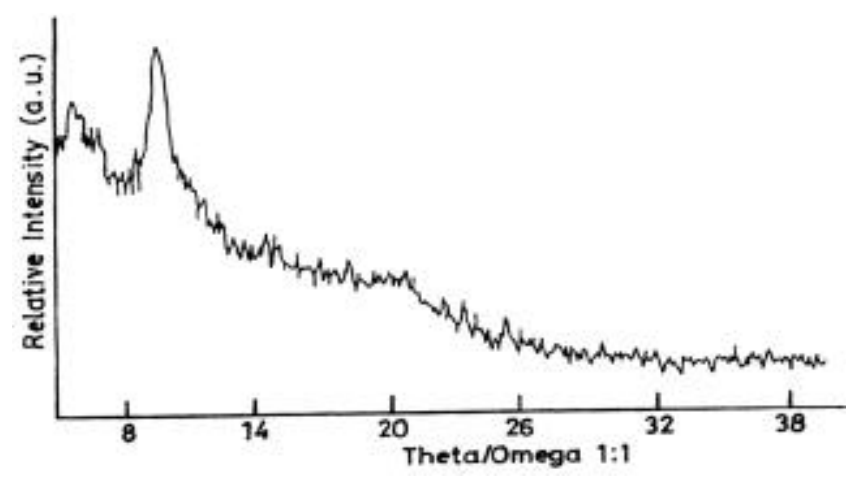

Figure 2. XRD pattern of pure polyvinyl alcohol.

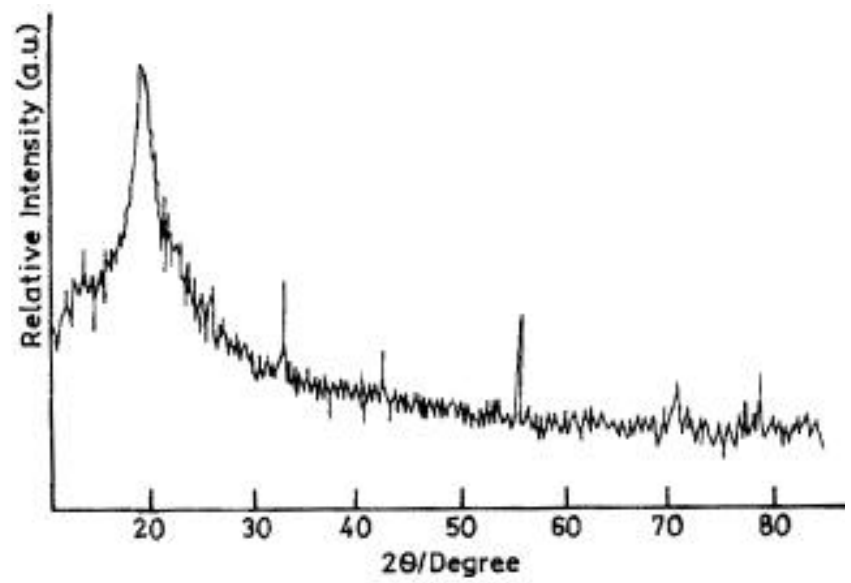

Figure 3. XRD pattern of as synthesized $\mathrm{MoO}_{3}-\mathrm{PVA}$ composite.

\subsection{Infrared study}

FTIR studies have been performed to understand the possible chemical interactions between $\mathrm{MoO}_{3}$ and PVA. Table 1 gives vibrational frequencies of as synthesized $\mathrm{MoO}_{3}$ and its PVA composite. Two peaks at 440 and $555 \mathrm{~cm}^{-1}$ correspond to metal-oxygen $(\mathrm{M}-\mathrm{O})$ bond. The bands in the range of $3600-3100 \mathrm{~cm}^{-1}$ (relating to antisymmetric and symmetric $\mathrm{OH}$ stretching) are observed in the present case and might be assigned to water of hydration. Hydrates also absorb in the region $1670-1600 \mathrm{~cm}^{-1}$ (relating to $\mathrm{OH}$ bending) (Miller and Walkins 1952). This latter band can be taken as another important means to identify water of crystallization and it has been found very useful in the elucidation of crystal structure. A broad peak observed at $1600 \mathrm{~cm}^{-1}$ in the present case corresponds to bending mode. The clear observation is because of some additional peaks and shifts in frequencies as compared to pure $\mathrm{MoO}_{3}$. We also observe peaks below $1000 \mathrm{~cm}^{-1}$ due to Mo-O vibrations. This confirms the presence of $\mathrm{MoO}_{3}$ particles present as dispersed materials inside the polyvinyl alcohol matrix.

\subsection{Thermal study}

Figure 6 shows the DSC trace of pure polyvinyl alcohol. In this trace there are two endothermic peaks observed at $100^{\circ} \mathrm{C}$ and $190^{\circ} \mathrm{C}$. The first endothermic peak corresponds to the removal of hydrated water and the second endothermic peak corresponds to glass transition of polyvinyl alcohol. After that there is a broad endo/exo peak observed due to decomposition of the sample. The end temperature of this could not be determined because of experimental limitation.

Figure 7 shows the DSC trace of $\mathrm{MoO}_{3}$-PVA composite. From this trace we observe the presence of two
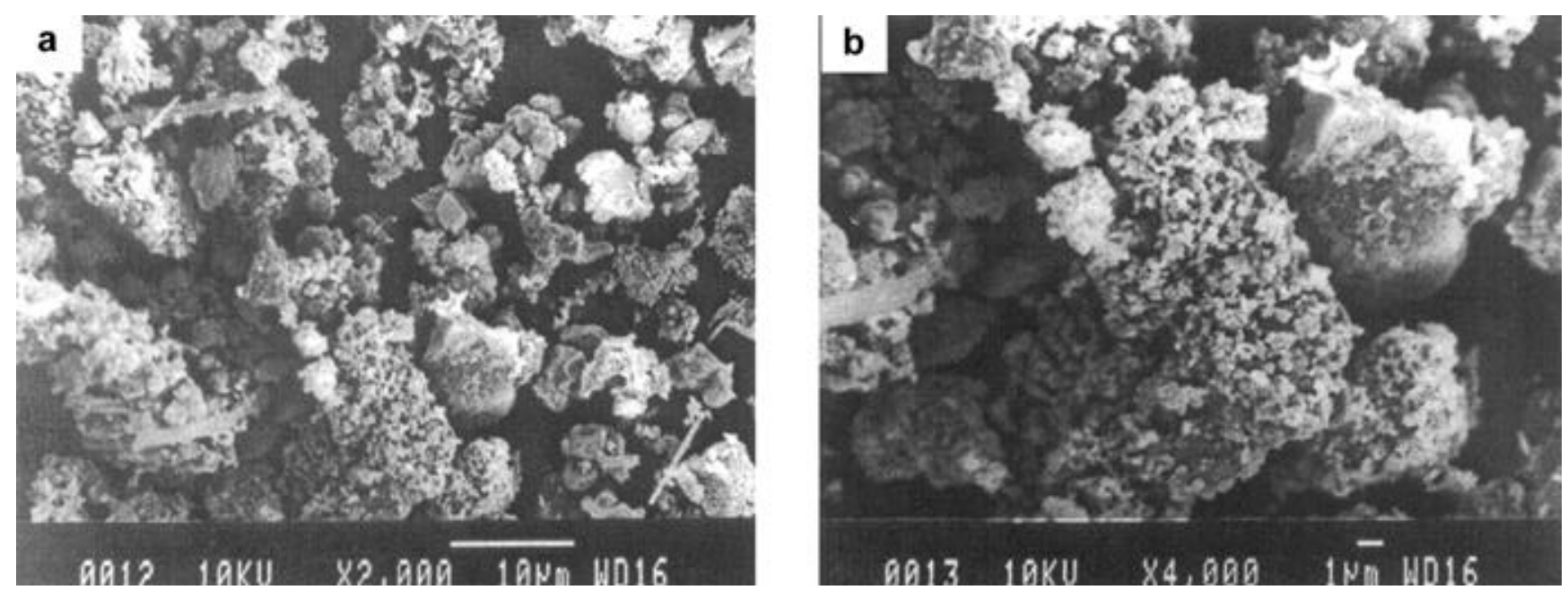

Figure 4. (a-b) SEM of as synthesized $\mathrm{MoO}_{3}$ at low and high magnifications. 

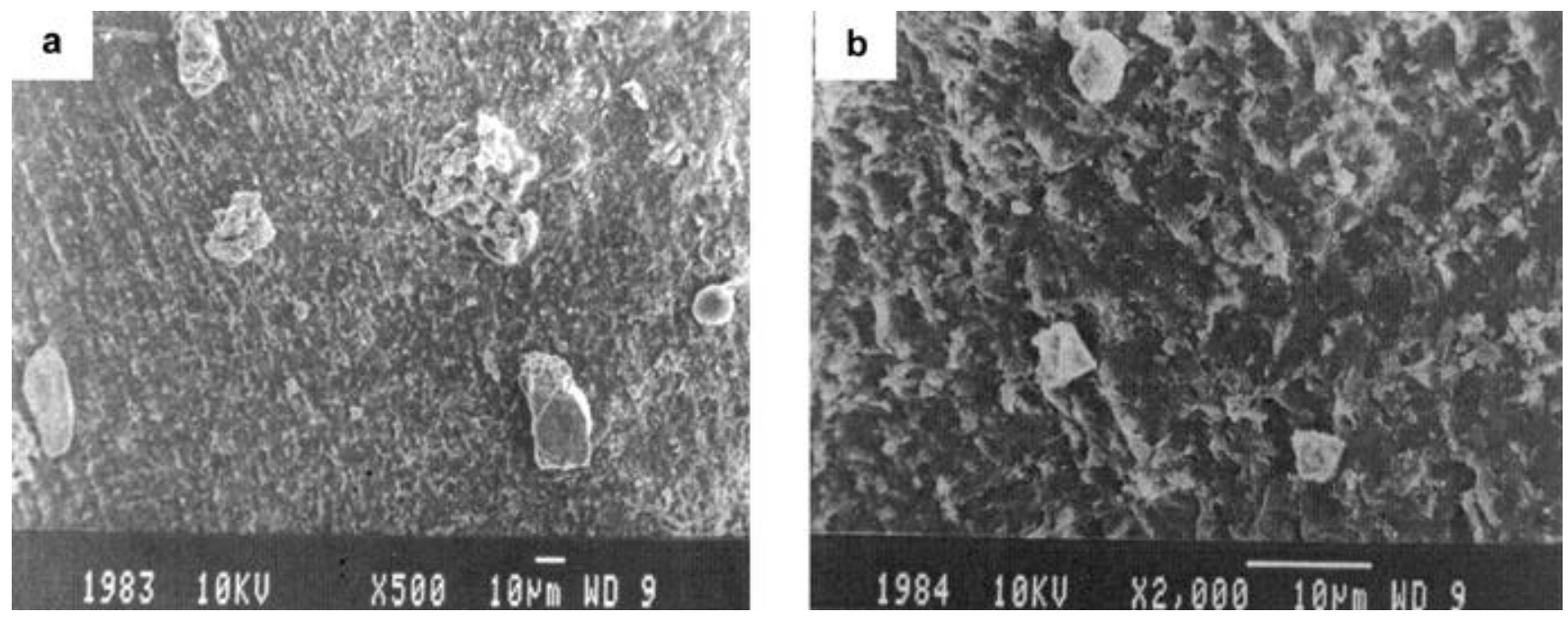

Figure 5. (a-b) SEM of as synthesized $\mathrm{MoO}_{3}-\mathrm{PVA}$ composite at low and high magnifications.

Table 1. Vibrational frequencies of as synthesized $\mathrm{MoO}_{3}$ and its composite with polyvinyl chloride.

\begin{tabular}{lcc}
\hline Sl. no. & $\begin{array}{c}\text { Vibrational frequencies } \\
\text { of } \mathrm{MoO}_{3}\end{array}$ & $\begin{array}{c}\text { Vibrational frequencies of } \\
\mathrm{MoO}_{3}-\mathrm{PVA} \text { composite }\end{array}$ \\
\hline 1 & 440 & 452 \\
2 & 555 & 560 \\
3 & 1600 & 1155 \\
4 & 3250 & 1505 \\
5 & - & 2000 \\
6 & - & 2610 \\
7 & - & 2905 \\
\hline
\end{tabular}

*All values are in $\mathrm{cm}^{-1}$.

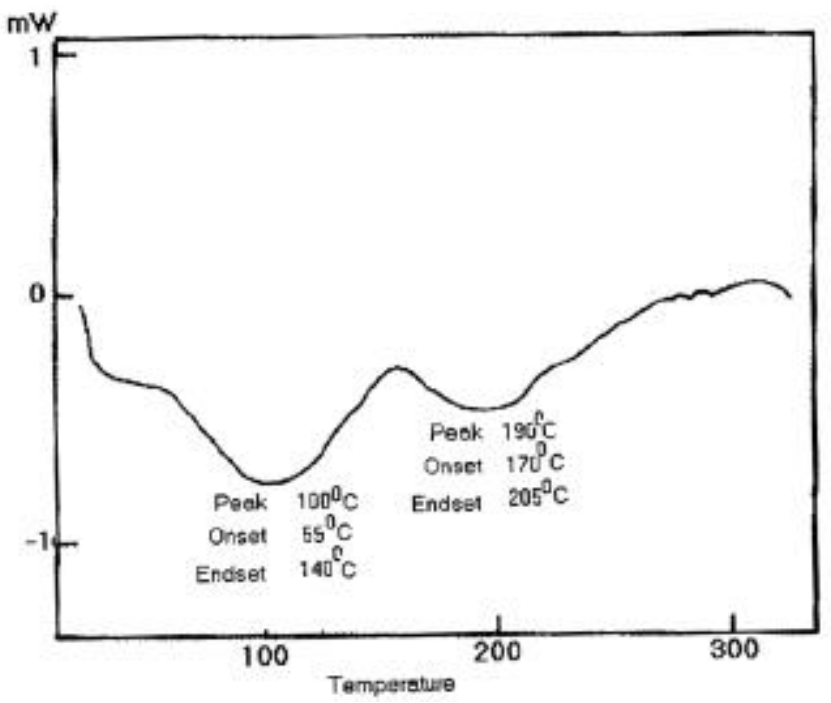

Figure 6. DSC of pure polyvinyl alcohol.

endothermic peaks, which are widely separated and shifted towards high temperature when compared with that of pure polyvinyl alcohol. The first endothermic peak at

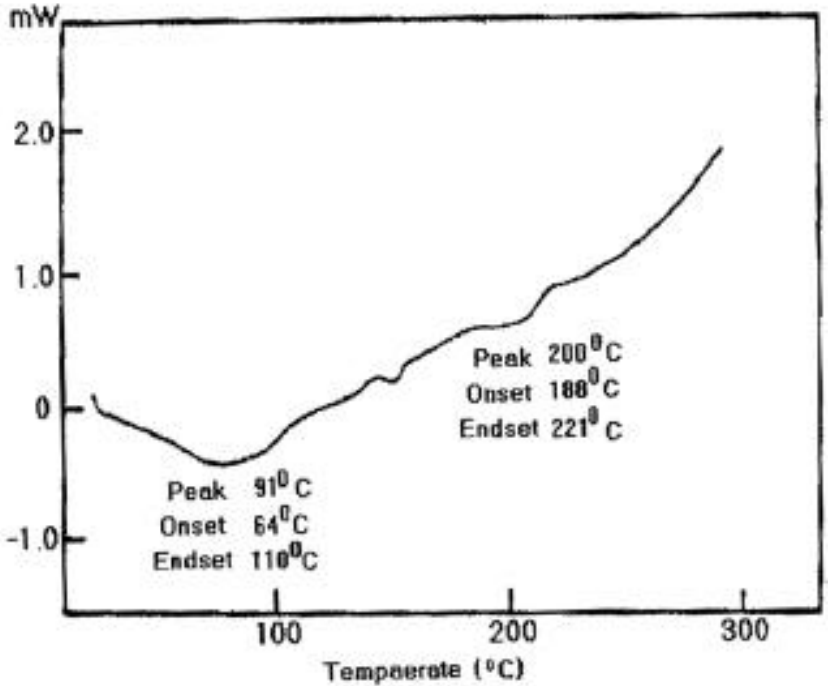

Figure 7. DSC of as synthesized $\mathrm{MoO}_{3}$-PVA composite.

$91^{\circ} \mathrm{C}$ corresponds to removal of hydrated water and second endothermic peak at $200^{\circ} \mathrm{C}$ is due to glass transition temperature, $T_{\mathrm{g}}$, of polyvinyl alcohol. On comparing both the thermograms, we observe a shift of $10^{\circ} \mathrm{C}$ temperature in glass transition temperature of polymer composite compared to the pure polyvinyl alcohol. The shift in $T_{\mathrm{g}}$ to higher temperature possibly indicates better chain entanglement of the polymer with the metal oxide. A detailed study with varying compositions of the $\mathrm{MoO}_{3}$ content is under progress, which then will help in confirming the chain entanglement of the polymer matrix with $\mathrm{MoO}_{3}$.

\section{Conclusions}

The combustion method for the synthesis of $\mathrm{MoO}_{3}$ is simple and easy to scale up; also this method may be 
applied for the synthesis of other layered ceramic materials. The distribution of $\mathrm{MoO}_{3}$ particles is found to be uniform throughout the polyvinyl alcohol matrix giving a crystalline and increased thermal stability for the films. Better chain entanglement of the polymer with $\mathrm{MoO}_{3}$ was noticed with increase in the glass transition temperature when compared to pure polyvinyl alcohol.

\section{References}

Castro C, Millan A and Palocio F 2000 J. Mater. Chem. 101945

Cheng K B, Ramakrishna S and Lee K C 2000 Composites Part A 311039

ChungChen Y and Huang C 2002 Mater. Sci. Engg. A334 250

Figlarz M 1989 Prog. Solid State Chem. 191

Gopalakrishnan J 1995 Chem. Mater. 71265

Govindraj B, Sastry N V and Venkataraman A 2004a J. Appl. Polym. Sci. 921527

Govindraj B, Sastry N V and Venkataraman A 2004b J. Appl. Polym. Sci. 93778

Harish Bhat M, Chakravarthy B P, Ramakrishnan P A, Levasseur A and Rao K J 2000 Bull. Mater. Sci. 23461

Helen G and Kamath P V 2000 Chem. Mater. 121195

Jungk H O and Feldmann C 2000 J. Mater. Res. 152244

Lagashetty A, Havanoor V and Venkataraman A 2002 Paper presented at the international symposium on 'Recent advances in inorganic materials' IIT, Mumbai

Lagashetty A, Mallikarjuna N N and Venkataraman A 2003 Indian J. Chem. Technol. 1063
Lee J G, Park J Y and Kim C S 1998 J. Mater. Sci. 333965

Mallikarjuna N N and Venkataraman A 2001 Indian J. Engg. \& Mater. Sci. 8303

Mallikarjuna N N and Venkataraman A 2003 Talanta 60139

Mallikarjuna N N, Govindraj B, Lagashetty A and Venkataraman A 2003a J. Therm. Anal. \& Cal. 71915

Mallikarjuna N N, Lagashetty A and Venkataraman A 2003b $J$. Therm. Anal. \& Cal. 74819

Mallikarjuna N N, Venkataraman A and Aminabhavi T M 2004 J. Appl. Polym. Sci. 942551

Miller F A and Walkins C H 1952 J. Anal. Chem. 241253

Rao C N R 1994 Chemical approaches to the synthesis of inorganic materials (New Delhi: Wiley Eastern Limited)

Rao K J, Vaidhyanathan B, Ganguli M and Ramakrishnan P A 1999a Chem. Mater. 1182

Rao K J, Ramakrishnan P A and Gadagkar R 1999b J. Solid State Chem. 148100

Richard J, Spontak P and Patel N P 2000 Current Opinion Colloid Interf. Sci. 5334

Shull R D and Bennett L H 1992 Nanostructured Mater. 183

Sinha R 2002 Outlines of polymer technology (New Delhi: Prentice Hall of India Private Limited) p. 93

Venkataraman A, Hiremath V A, Date S K and Kulkarni S D 2001 Bull. Mater. Sci. 24617

Xiao T D, Zhang Y D, Strutt P R, Budric J I, Mohan K and Gonsalves K E 1993 Nanostructured Mater. 2285

Yurekli K, Krishnamoorti R, Tse M F, Mcelrath K O, Tsou A H and Wang H C 2001 J. Polym. Sci. Part B Polym. Phys. 39 256

Zdujic M, Jovalekic C, Karanovic Lj, Mitric M, Poleti D and Skala D 1998 Mater. Sci. Engg. A245 109 